\title{
Níveis de arginina e lisina digestíveis na dieta de frangos de corte na fase pré- inicial
}

\author{
José Henrique Stringhini ${ }^{1,4}$, Cícero Peres da Cruz ${ }^{2,4}$, Mônica Schaitl Thon ${ }^{2,4}$, Maria Auxiliadora \\ Andrade $^{3,4}$, Nadja Susana Mogyca Leandro ${ }^{1,4}$, Marcos Barcellos Café1,4
}

\footnotetext{
${ }^{1}$ Departamento de Produção Animal, Escola de Veterinária da Universidade Federal de Goiás, caixa postal 131, 74001-970, Goiânia, Goiás. 2 Mestrando em Ciência Animal pela Escola de Veterinária da Universidade Federal de Goiás, caixa postal 131, 74001-970, Goiânia, Goiás.

${ }^{3}$ Departamento de Medicina Veterinária, Escola de Veterinária da Universidade Federal de Goiás, caixa postal 131, 74001-970, Goiânia, Goiás.
} ${ }^{4}$ Bolsista do CNPq.

RESUMO - Avaliaram-se o desempenho, a digestibilidade e retenção de nutrientes e o desenvolvimento de órgãos de frangos de corte na fase pré-inicial (1 a 7 dias de idade) alimentados com dietas contendo diferentes níveis de lisina e arginina digestíveis. Um total de 352 pintos AgRoss machos foram distribuídos em um arranjo fatorial $2 \times 3$ composto de dois níveis de lisina digestível (1,056 e $1,305 \%)$ e três níveis de arginina digestível (1,305; 1,459 e 1,613\%), com quatro repetições de 11 aves. O ganho de peso diário não foi afetado pelas dietas, porém, houve efeito quadrático dos níveis de arginina sobre a conversão alimentar de 1 a 7 dias e de 1 a 21 dias de idade e efeito linear sobre o consumo de alimento no período de 1 a 21 dias de idade. O peso relativo do proventrículo + moela reduziu à medida que o nível de arginina digestível aumentou para o nível de 1,305\% de lisina digestível aos 14 dias de idade. Os valores do coeficiente de digestibilidade dos nutrientes e retenção de MS e N foram melhores nos menores níveis de lisina digestível. Os níveis de 1,40 a 1,46\% de arginina digestível podem ser utilizados em dietas para frangos de corte na fase pré-inicial, mas, à medida que aumenta o conteúdo de lisina da dieta, deve-se manter a relação desse aminoácido com arginina.

Palavras-chave: desempenho, desenvolvimento do trato digestório, digestibilidade, nutrição pós-eclosão

\section{Digestible arginine and lysine levels in pre-starter broiler diet}

\begin{abstract}
The performance, digestibility and retention of nutrients and the development of digestive organs of broiler chicks in pre-starter diet (1 to 7 days old) fed diets with different levels of digestible lysine and arginine. A total of 352 day old AgRoss male chicks was allotted to 2 × 3 factorial arrangement (level of digestible lysine [1.056 and 1.305\%] and levels of digestible arginine [1.305, 1.459 and 1.613\%] and four replicates of 11 chicks. Daily weight gain was not affected by diets but there was a quadratic effect of arginine levels on feed:gain ratio from 1 to 7 and from 1 to 21 days old and linear effect on feed intake from 1 to 21 days old. As for organs development, it was observed a reduction for the relative weight of proventriculus + gizzard as arginine level increased for the $1.305 \%$ digestible lysine level at 14 days of age The digestibility coefficients of nutrients and retention of DM and $\mathrm{d} N$ values were better for the low digestible lysine levels. The levels from 1.40 to 1.46 of digestible arginine could be used for pre-starter phase diets of broiler chicks, however as lysine content increase in the diet its relation with arginine should be adjusted.
\end{abstract}

Key Words: digestibility, digestive organs development, nutrition after hatch, performance

\section{Introdução}

A seleção genética impôs às aves elevada velocidade de crescimento, o que exige aporte de nutrientes digestíveis para adequado desenvolvimento fisiológico e dos sistemas imunológico e esquelético da ave (Penz Jr. \& Renz, 2003). A fase pré-inicial representa de 20 a $30 \%$ do período total de vida da ave e corresponde dos 7 aos 10 dias de idade, quando deve ser fornecida dieta específica (Toledo et al., 2001; Araújo, 2003).

Vários fatores influenciam o crescimento precoce no período pós-eclosão, entre eles, o conteúdo de resíduos do saco vitelínico, a ingestão de alimento e água, os níveis de enzimas pancreáticas e intestinais, a área de superfície intestinal e a digestibilidade global de nutrientes (Moran Jr., 1985; Dibner, 1996). Neste período, as aves apresentam dificuldade em digerir e absorver nutrientes como lipídios, em virtude da imaturidade de circulação enterohepática de sais biliares e da grande dificuldade de termorregulação, decorrente do consumo elevado de proteína para produzir calor metabólico (Penz \& Vieira, 1998).

Existem pontos de vista divergentes sobre níveis nutricionais na fase pré-inicial e sobre a forma física, o tempo e a quantidade da dieta específica fornecida. Apesar 
dessas divergências, informações recentes sobre necessidades nutricionais na fase pré-inicial indicaram valores diferentes dos observados para aves mais velhas, porém, as informações disponíveis são escassas (Penz \& Renz, 2003; Stringhini et al., 2003; Rostagno, 2005).

D’Mello (1993) sugeriu que os aminoácidos limitantes para aves são metionina, lisina e triptofano, nesta ordem. A arginina é um dos aminoácidos disponibilizados para estudos de exigências nutricionais, mas sua suplementação em dietas ainda não é freqüente, embora seja considerada aminoácido essencial e com importantes funções em mamíferos e aves (Edmonds et al., 1985; Cynober et al., 1995; Mendes et al., 1997). Gadelha (2004) comprovou que a suplementação de arginina em níveis elevados (2,06\%) pode contribuir para reduzir problemas de perna de aves em estresse por calor se mantidas relações de 1,05 a 1,1 com lisina.

Ajinomoto (2004) indicou níveis de lisina digestível de pelo menos $1,20 \%$ para a fase pré-inicial, superiores ao recomendado para a fase inicial. Entretanto, sabe-se que existe importante relação entre lisina e arginina na nutrição de frangos e que é necessário ajustar seus níveis (Hurwitz et al., 1998; Chamruspollert et al., 2002b, 2004), porém, em experimentos descritos anteriormente por Ajinomoto (1990), a suplementação de arginina teve pouco efeito sobre o crescimento e promoveu pequena melhora na eficiência alimentar. As necessidades de lisina para ótimo desempenho da ave jovem aumentaram com o nível de proteína, independentemente do conteúdo de arginina.

A digestibilidade dos nutrientes é afetada pela idade das aves. Batal \& Parsons (2002a, 2003) verificaram que dietas fornecidas logo após eclosão (1으 e $2^{\underline{0}}$ dia de idade) e na segunda semana de idade ( $11^{\circ}$ a $14^{\circ}$ dia de idade) apresentaram resultados diferentes para energia metabolizável (2.970 e $3.430 \mathrm{kcal} \mathrm{EM} / \mathrm{kg}$ ) e digestibilidade da lisina (78 e 89\%).

Batal \& Parsons (2002b) observaram que dietas à base de aminoácidos, em comparação a dietas à base de milho e farelo de soja, interferiram nos pesos absoluto e relativo de órgãos (intestino delgado, pâncreas, fígado, moela e proventrículo) no período entre 7 e 21 dias de idade.

O objetivo neste experimento foi avaliar o desempenho, a digestibilidade de nutrientes e o desenvolvimento de órgãos de frangos de corte na fase pré-inicial alimentados com dietas com diferentes níveis de lisina e arginina digestíveis.

\section{Material e Métodos}

O experimento foi conduzido no Aviário experimental da Escola de Veterinária da Universidade Federal de Goiás, em Goiânia, no período de março a agosto de 2004.
Foram utilizados pintos de corte AgRoss machos vacinados no incubatório contra Marek. Foram utilizadas oito baterias de aço galvanizado, equipadas com comedouros e bebedouros lineares e bandejas metálicas para retirada das excretas. Cada bateria continha cinco andares com divisões de $0,33 \times 0,50 \mathrm{~m}$ totalizando 40 unidades experimentais. Utilizou-se uma lâmpada incandescente de $60 \mathrm{~W}$ por andar até os 14 dias para aquecimento das aves. O manejo até 21 dias incluiu a limpeza diária dos bebedouros, a troca de água, o abastecimento de comedouros duas vezes ao dia, a verificação da temperatura e o manejo das cortinas.

As aves foram uniformizadas de acordo com o peso no início do experimento e distribuídas em um arranjo fatorial $2 \times 3$ composto de dois níveis de lisina digestível (1,056 e 1,305\%) e três níveis de arginina digestível $(1,305 ; 1,459$ e $1,613 \%)$, com quatro repetições de 11 aves. Foram avaliados o peso médio, o consumo de ração, o índice de conversão alimentar e a taxa de mortalidade das aves de 1 a 7 e de 1 a 21 dias de idade. Apenas para análise estatística, os dados de mortalidade foram transformados em arco seno.

Todas as dietas experimentais (Tabela 1) foram isoenergéticas e isonutritivas, à base de milho e farelo de soja. As dietas foram formuladas para atender às exigências nutricionais descritas por Rostagno et al. (2000). Tanto a arginina como a lisina sintética foram suplementadas em substituição ao amido de mandioca. As dietas foram fornecidas do $1 \underline{0}$ ao $7 \underline{0}$ dia de idade e, na fase inicial ( 8 a 21 dias), todas as aves consumiram a mesma dieta (Tabela 1 ).

As aves foram submetidas a um período de adaptação nos três primeiros dias e, do 4 으 ao 70 dia, foi realizada a coleta total de excretas. As excretas e as dietas experimentais foram analisadas para determinação do teor de MS e de nitrogênio total, segundo metodologia proposta por Silva \& Queiroz (2002). Os dados foram tabulados para determinação do coeficiente de digestibilidade (CD), calculado pela fórmula proposta por Matterson et al. (1965), e da retenção (RET) de nutrientes, como descrito por Noy \& Sklan (2002).

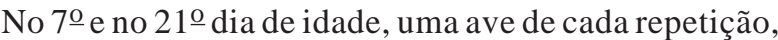
representando a média de peso da unidade experimental, foi selecionada e transportada ao Laboratório de Doenças das Aves do Departamento de Medicina Veterinária na EV/ UFG, onde foi sacrificada por deslocamento cervical. Foram avaliados o comprimento intestinal e os pesos do esôfago + inglúvio, proventrículo + moela, fígado, pâncreas e intestino. Esses valores foram tabulados e serviram como base para o cálculo do peso relativo dos órgãos em relação ao peso vivo.

Os dados foram submetidos à análise de variância e à análise de regressão polinomial, adotando-se as análises 
Tabela 1 - Composições percentual e nutricional das dietas experimentais

Table 1 - Percentage and nutritional compositions of the experimental diets

\begin{tabular}{lcc}
\hline $\begin{array}{l}\text { Ingrediente } \\
\text { Ingredient }\end{array}$ & $\begin{array}{c}\text { Pré-inicial } \\
\text { (basal) } \\
\text { Pre-starter } \\
\text { (basal) }\end{array}$ & $\begin{array}{c}\text { Inicial } \\
\text { (8 a 21 dias) } \\
\text { Starter } \\
\text { (8 to 21 days) }\end{array}$ \\
\hline Milho (Corn) & 54,754 & 59,409 \\
Farelo de soja (Soybean meal) & 24,000 & 37,096 \\
Soja integral tostada & 16,000 & - \\
Toasted full-fat soybean & & \\
Sal comum (Salt) & 0,400 & 0,272 \\
Amido mandioca & 1,000 & - \\
Cassava starch & & \\
Calcário calcítico (Limestone) & 1,000 & 0,952 \\
Fosfato bicálcico (Dicalcium phosphate) & 2,000 & 1,970 \\
Suplemento vitamínico-mineral & 0,180 & 0,150 \\
Mineral and vitamin premix & & \\
Bicarbonato de sódio & 0,208 & - \\
Sodium bicarbonate & & \\
DL-metionina (DL-methionine) & 0,200 & - \\
Lisina-HCl (Lysine HCl) & 0,033 & - \\
Cloreto de colina (Choline chloride) & 0,100 & - \\
Aditivos (Additives) & 0,125 & - \\
\hline
\end{tabular}

Composição nutricional calculada

Calculated composition

\begin{tabular}{|c|c|c|}
\hline $\mathrm{PB}(C P)(\%)$ & 21,576 & 21,1 \\
\hline EM (ME) (kcal/kg) & 3.000 & 3.000 \\
\hline Са $(\%)$ & 0,957 & 0,966 \\
\hline P disponível (Available P) (\%) & 0,501 & 0,484 \\
\hline Lisina total (Total lysine) (\%) & 1,240 & 1,200 \\
\hline Lisina digestível (Digestible lysine) (\%) & 1,056 & - \\
\hline Metionina total $(\%)$ & 0,537 & - \\
\hline \multicolumn{3}{|l|}{ Total methionine } \\
\hline Metionina digestível (\%) & 0,496 & - \\
\hline \multicolumn{3}{|l|}{ Digestible lysine } \\
\hline Aminoácidos sulfurados totais (\%) & 0,891 & 0,794 \\
\hline \multicolumn{3}{|l|}{ Total sulphur amino acids } \\
\hline Aminoácidos sulfurados digestível (\%) & 0,780 & - \\
\hline \multicolumn{3}{|l|}{ Digestible sulphur amino acids } \\
\hline Treonina total (Total threonine) (\%) & 0,836 & 0,860 \\
\hline Treonina digestível (\%) & 0,695 & - \\
\hline \multicolumn{3}{|l|}{ Digestible threonine } \\
\hline Triptofano total (\%) & 0,280 & 0,296 \\
\hline \multicolumn{3}{|l|}{ Total thryptophan } \\
\hline Triptofano digestível (\%) & 0,236 & - \\
\hline \multicolumn{3}{|l|}{ Digestible thriptophan } \\
\hline Arginina total $(\%)$ & 1,459 & - \\
\hline \multicolumn{3}{|l|}{ Total arginine } \\
\hline Arginina digestível (\%) & 1,305 & \\
\hline \multicolumn{3}{|l|}{ Digestible threonine } \\
\hline $\mathrm{Na}(\%)$ & 0,247 & 0,154 \\
\hline $\mathrm{Na}+\mathrm{K}+\mathrm{Cl}(\mathrm{Meq} / 100 \mathrm{~g})$ & 248,415 & \\
\hline
\end{tabular}

1 Suplemento vitamínico para frangos - Fase inicial (vitamin supplement for broilers - initial phase): vit. A - 3.125.000 UI; vit. D3 - 550.000 UI; vit. E $3.750 \mathrm{mg}$; vit. K3 - $625 \mathrm{mg}$; vit. B1 - $250 \mathrm{mg}$; vit. B2 - $1.125 \mathrm{mg}$; vit. B6 - 250 mg; vit. B12 - 3.750 mg; niacina (niacin) - 9.500 mg; pantotenato de cálcio (calcium pantothenate), $3.750 \mathrm{mg}$; ác. fólico (folic acid) - $125 \mathrm{mg}$; DL-metionina (DL-methionine) - $350.000 \mathrm{mg}$; cloreto de colina 50\% $150.000 \mathrm{mg}$; promotor de crescimento (growth promoter) - $12.500 \mathrm{mg}$; coccidiostático - $15.000 \mathrm{mg}$; Se - $50 \mathrm{mg}$; antioxidante (antioxidant) $2.500 \mathrm{mg}$; veículo q.s.p., $1000 \mathrm{~g}$. Suplemento mineral (Mineral supplement): $\mathrm{Fe}-100.000 \mathrm{mg}$; Cu - 16.000 mg; Zn - 100.000 mg; I 1500 mg.

2 Níveis de arginina crescentes calculados adicionando 0,1\% de L-arginina nas dietas em substituição ao amido de mandioca (Arginine levels were calculated adding $0.1 \%$ of $L$-arginine in substitution to cassava starch).

${ }^{3}$ Avilamicina (Avilamycin), Olaquindox (Olaquindox), sal monossódico de monensina (monensin, monosodium salt), BHT. estatísticas para os níveis de arginina digestível e o teste F para níveis de lisina digestível, realizados no programa computacional SAEG (UFV, 2000).

\section{Resultados e Discussão}

A suplementação de arginina digestível influenciou o consumo no período de 1 a 21 dias de idade $(\mathrm{P}<0,05)$ e a conversão alimentar $(\mathrm{P}<0,05)$ nos períodos de 1 a 7 dias e de 1 a 21 dias de idade (Tabela 2), o que está de acordo com os resultados obtidos por Gadelha (2004), que testou níveis de arginina digestível de 1,29 ou 2,06\% e observou que a suplementação de arginina em relações arginina:lisina de 1,69 na dieta na fase inicial melhorou o desempenho das aves. Resultados positivos com suplementação de arginina foram observados por Chamruspollert et al. (2004) nos níveis de 1,52 e 2,52\%, mas pioraram com 3,52 \% na dieta inicial (1 a 21 dias de idade).

As altas concentrações de lisina na dieta podem influenciar a atuação e as exigências de arginina, que, por sua vez, interferem nas exigências de metionina, afetando o desenvolvimento das aves (Chamruspollert et al., 2002a,b).

Ao contrário do observado neste experimento, Kidd et al. (2001) verificaram que níveis semelhantes aos recomendados pelo NRC (1994) para a fase inicial $(1,25 \%)$ são suficientes para suportar o bom crescimento e manter normais as funções imunológicas da ave. Neste sentido, Chamruspollert et al. (2002a,b) constataram que altos níveis de arginina na dieta inicial (1,52, 2,52 e 3,52\%) reduziram o desempenho de frangos de corte de 1 a 14 dias de idade. Esses autores observaram interação tripla arginina $\times$ lisina $\times$ metionina e interferência no metabolismo da arginase renal e na creatina muscular, o que indica que o equilíbrio entre esses aminoácidos deve ser preservado.

Os níveis de arginina e lisina digestíveis não influenciaram $(\mathrm{P}>0,05)$ o ganho de peso, o peso e as dimensões de órgãos (Tabelas 3 e 4 ), porém afetaram $(\mathrm{P}<0,05)$ o peso da moela. Hurwitz et al. (1998) sugeriram que aves alimentadas com dietas suplementadas com arginina em baixos conteúdos de proteína são eficientes em reduzir a deposição de gordura e melhorar a eficiência digestiva.

Batal \& Parsons (2002b) verificaram que dietas formuladas à base de aminoácidos digestíveis, em comparação a dietas de milho e farelo de soja formuladas com base no conteúdo de proteína total, interferiram no peso absoluto de intestino delgado, pâncreas, fígado, moela e proventrículo aos 7 e aos 21 dias de idade. Quando considerado o peso relativo (relação com ganho de peso), os valores foram inferiores aos obtidos aos 7 dias de idade, mas semelhantes aos encontrados aos 21 dias de idade. 
Tabela 2 - Ganho de peso médio (GPM), consumo de alimento (CR) e conversão alimentar (CA) nos períodos de 1 a 7 dias e de 1 a 21 dias de idade obtidos com frangos alimentados com dietas com diferentes níveis de arginina (Arg) e lisina (Lys) digestíveis na fase pré-inicial

Table 2 - $\quad$ Average weight gain (MWG), feed intake (FI) and feed-to-gain ratio (FGR) from 1 to 7 days and from 1 to 21 days of age, obtained with broilers fed diets with different levels of digestible arginine (Arg) and lysine (Lys) in the pre-starter phase

\begin{tabular}{|c|c|c|c|c|c|c|}
\hline \multirow[t]{2}{*}{$\begin{array}{l}\text { Arginina digestível (\%) } \\
\text { Digestible arginine }\end{array}$} & \multicolumn{3}{|c|}{$\begin{array}{l}1 \text { a } 7 \text { dias } \\
1 \text { to } 7 \text { days }\end{array}$} & \multicolumn{3}{|c|}{$\begin{array}{l}1 \text { a } 21 \text { dias } \\
1 \text { to } 21 \text { days }\end{array}$} \\
\hline & $\begin{array}{l}\text { GPM (g) } \\
M W G, g\end{array}$ & $\begin{array}{l}\text { CR (g) } \\
\quad F I\end{array}$ & $\begin{array}{c}\text { CA }(\mathrm{g} / \mathrm{g})^{1} \\
F G R\end{array}$ & $\begin{array}{l}\text { GPM (g) } \\
M W G\end{array}$ & $\begin{array}{l}\text { CR (g) } \\
\quad F I\end{array}$ & $\begin{array}{c}\text { CA }(\mathrm{g} / \mathrm{g}) \\
F G R\end{array}$ \\
\hline 1,305 & 141,18 & 144,66 & $1,03 \mathrm{~b}$ & 656,85 & $1012,32 b$ & $1,54 \mathrm{~b}$ \\
\hline 1,459 & 128,98 & 148,26 & $1,16 \mathrm{a}$ & 625,11 & $1045,93 a$ & $1,68 a$ \\
\hline 1,613 & 134,31 & 148,64 & $1,11 \mathrm{ab}$ & 637,15 & $1048,11 \mathrm{a}$ & $1,65 \mathrm{a}$ \\
\hline \multicolumn{7}{|l|}{$\begin{array}{l}\text { Lisina digestível (\%) } \\
\text { Digestible lysine }\end{array}$} \\
\hline 1,056 & 135,45 & 150,16 & 1,12 & 633,10 & 1030,24 & 1,64 \\
\hline 1,305 & 134,20 & 144,22 & 1,08 & 646,31 & 1040,67 & 1,61 \\
\hline $\begin{array}{l}\text { Probabilidade (\%) } \\
\text { Probability }\end{array}$ & 0,27 & 0,12 & 0,04 & - & 0,02 & 0,02 \\
\hline $\begin{array}{l}\text { Probability } \\
\text { Coeficiente de variação (\%) } \\
\text { Coefficient of variation }\end{array}$ & 8,725 & 6,148 & 9,945 & 5,893 & 2,736 & 6,673 \\
\hline
\end{tabular}

$\hat{Y}=-7,592+0,0117 x-0,000004 x^{2}, r^{2}=1,00$.

Tabela 3 - Comprimento intestinal (CInt) e pesos relativos do papo + esôfago (PEsof), proventrículo + moela (PMoe), fígado (PFig), intestino delgado (PID), peso intestino grosso (PIG) e pâncreas (PPanc) obtidos com frangos de corte aos 7 dias de idade alimentados na fase pré-inicial com dietas com diferentes níveis de arginina (Arg) e lisina (Lys) digestíveis

Table 3 - Intestinal length (IL), relative weight of crop + esophagus (CEW), proventriculus + gizzard (PGW), liver (LW), small intestine (SIW), gross intestine (GIW) and pancreas (PW) relative weight obtained with broilers at 7 days of age fed diets with different levels of digestible arginine (Arg) and lysine (Lys) in pre-starter phase

\begin{tabular}{|c|c|c|c|c|c|c|c|}
\hline $\begin{array}{l}\text { Arginina digestível (\%) } \\
\text { Digestible arginine }\end{array}$ & $\begin{array}{c}\text { CInt }(\mathrm{cm}) \\
I L\end{array}$ & $\begin{array}{l}\text { Pesof }(\%) \\
\text { CEW }\end{array}$ & $\begin{array}{l}\text { PMoe (\%) } \\
\quad P G W\end{array}$ & $\begin{array}{l}\text { Pfig (\%) } \\
\quad L W\end{array}$ & $\begin{array}{l}\text { PID (\%) } \\
\text { SIW }\end{array}$ & $\begin{array}{l}\text { PIG (\%) } \\
\text { GIW }\end{array}$ & $\begin{array}{c}\text { PPanc, \% } \\
P W\end{array}$ \\
\hline 1,305 & 94,06 & 1,23 & 7,78 & 4,16 & 6,78 & 1,93 & 0,55 \\
\hline 1,459 & 93,68 & 1,33 & 7,98 & 4,09 & 6,64 & 1,76 & 0,51 \\
\hline 1,613 & 93,68 & 1,36 & 8,15 & 4,24 & 6,95 & 1,65 & 0,51 \\
\hline \multicolumn{8}{|l|}{$\begin{array}{l}\text { Lisina digestível (\%) } \\
\text { Digestible lysine }\end{array}$} \\
\hline 1,056 & 92,04 & 1,27 & 7,76 & 4,22 & 6,78 & 1,74 & 0,54 \\
\hline 1,305 & 95,57 & 1,34 & 8,19 & 4,11 & 6,79 & 1,82 & 0,50 \\
\hline $\begin{array}{l}\text { Probabilidade (\%) } \\
\text { Probability }\end{array}$ & 0,16 & - & - & - & - & - & 0,31 \\
\hline $\begin{array}{l}\text { Coeficiente de variação (\%) } \\
\text { Coefficient of variation }\end{array}$ & 7,769 & 17,816 & 10,618 & 13,291 & 11,817 & 29,909 & 14,049 \\
\hline
\end{tabular}

Houve efeito da interação $(\mathrm{P}<0,05)$ dos níveis de lisina e de arginina sobre o peso da moela + proventrículo aos 14 dias de idade (Tabela 5). Houve redução para o peso relativo do proventrículo + moela comparando-se o nível de 1,305\% com 1,615\% de arginina digestível.

Esses resultados concordam parcialmente com o observado por Valério et al. (2003), que encontraram redução linear do peso da moela quando os níveis de lisina digestível foram aumentados.

Os coeficientes de digestibilidade não diferiram entre os níveis crescentes de arginina digestível na dieta (Tabela 6). Entretanto, o balanço e a retenção de nitrogênio pioraram por unidade de ganho de peso no maior nível de arginina utilizado, semelhante ao obtido por Stringhini et al. (2006) em experimento com dietas contendo níveis decrescentes de proteína e níveis constantes de lisina total (1,35\%).

Analisando as equações de regressão obtidas no experimento (Tabela 7), verificou-se que os níveis de arginina digestível sugeridos variam de 1,40 a 1,46\%, independentemente do nível de lisina digestível adotado. Os níveis de arginina digestível sugeridos são de 1,40 a 1,46\%, independentemente do nível de lisina digestível. Esses níveis são superiores aos determinados por Rostagno et al. (2000), de $1,290 \%$ e 1,200 nas bases total e digestível, e aos valores 
Tabela 4 - Comprimento intestinal (CInt) e pesos relativos do papo + esôfago (PEsof), proventrículo + moela (PMoe), fígado (PFig), intestino delgado (PID), intestino grosso (PIG) e pâncreas (PPanc) de frangos de corte aos 14 dias de idade alimentados na fase préinicial com dietas com diferentes níveis de arginina (Arg) e de lisina (Lys) digestíveis

Table 4 - Intestinal length (IL) and relative weights of crop + esophagus (CEW), proventriculus + gizzard (PGW), liver (LW), small intestine (SIW), gross intestine (GIW) and pancreas (PW) obtained with broilers at 14 days of age fed diets with different levels of digestible arginine (Arg) and lysine (Lys) in pre-starter phase

\begin{tabular}{|c|c|c|c|c|c|c|c|}
\hline $\begin{array}{l}\text { Arginina digestível (\%) } \\
\text { Digestible arginine }\end{array}$ & $\begin{array}{c}\text { CInt }(\mathrm{cm}) \\
I L\end{array}$ & $\begin{array}{l}\text { Pesof (\%) } \\
\text { CEW }\end{array}$ & $\begin{array}{l}\text { PMoe (\%) } \\
\quad P G W\end{array}$ & $\begin{array}{l}\text { Pfig (\%) } \\
\qquad W\end{array}$ & $\begin{array}{l}\text { PID (\%) } \\
\text { SIW }\end{array}$ & $\begin{array}{l}\text { PIG (\%) } \\
\text { GIW }\end{array}$ & $\begin{array}{c}\text { PPanc }(\%) \\
P W\end{array}$ \\
\hline 1,305 & 110,81 & 1,01 & 4,90a & 4,19 & 4,56 & 0,93 & 0,41 \\
\hline 1,613 & 112,59 & 0,99 & $4,42 b$ & 3,99 & 4,42 & 0,91 & 0,38 \\
\hline
\end{tabular}

Lisina digestível (\%)

Digestible lysine

\begin{tabular}{|c|c|c|c|c|c|c|c|}
\hline 1,056 & 111,75 & 0,96 & 4,54 & 4,14 & 4,52 & 0,90 & 0,38 \\
\hline 1,305 & 111,04 & 0,98 & 4,74 & 3,94 & 4,37 & 1,06 & 0,41 \\
\hline Probabilidade (\%) & - & 0,19 & 0,04 & & - & - & - \\
\hline Probability & & & & & & & \\
\hline
\end{tabular}

Tabela 5 - Peso relativo do proventrículo + moela (PMoe) obtidos com frangos aos 14 dias de idade alimentados na fase pré-inicial com dietas com diferentes níveis de arginina e de lisina

Table 5 - $\quad$ Relative weight of proventriculus + gizzard (PGW) obtained with broilers at 14 days of age fed diets with different levels of digestible arginine (Arg) and lysine (Lys) in pre-starter phase

\begin{tabular}{lcr}
\hline $\begin{array}{l}\text { Arginina digestível (\%) } \\
\text { Digestible arginine }\end{array}$ & \multicolumn{2}{c}{$\begin{array}{c}\text { Lisina digestível (\%) } \\
\text { Digestible lysine }\end{array}$} \\
\hline & 1,056 & 1,305 \\
1,305 & $4,50 \mathrm{bA}$ & $5,30 \mathrm{aA}$ \\
1,459 & $4,60 \mathrm{aA}$ & $4,60 \mathrm{aB}$ \\
1,613 & $4,53 \mathrm{aA}$ & $4,31 \mathrm{aB}$ \\
Probabilidade (\%) & 0,02 & \\
Probability & & \\
Coeficiente de variação (\%) & 8,604 \\
Coefficient of variation & & \\
\hline
\end{tabular}

Letras minúsculas iguais indicam não haver diferença $(P>0,05)$ dos níveis de Lys dentro dos níveis de arginina digestível.

Médias, com letras maiúsculas iguais indicam não haver diferença $(P>0,05)$ dos níveis de arginina digestível dentro dos níveis de Lys.

Equal minor letters did not indicate statistical significant difference $(P>0.05)$ of digestible lysine levels within digestible arginine levels.

Means followed by capital letters did not indicate statistical significant difference $(P>0.05)$ of digestible arginine levels within digestible lysine levels.

estimados para a fase pré-inicial, de 1,328 e 1,236\%, respectivamente. Os resultados desse experimento corroboram os propostos por Rostagno (2005), de 1,367 e 1,431\% de arginina digestível.

Níveis altos de arginina em altas relações com lisina, calculados na base de aminoácidos digestíveis, promoveram respostas satisfatórias de desempenho, como demonstrado por Gadelha (2004). Kidd et al. (2001) verificaram que
Tabela 6 - Coeficiente de digestibilidade da MS e PB e retenção de MS e $\mathrm{N}$ em frangos de corte de 4 a 7 dias de idade alimentados na fase pré-inicial com dietas com diferentes níveis de arginina e lisina

Table 6 - Coefficient of digestibility of DM and CP and retention (RET) of $D M$ and $N$ obtained with broilers from 4 to 7 days of age fed diets with different levels of digestible arginine (Arg) and lysine (Lys) in pre-starter phase

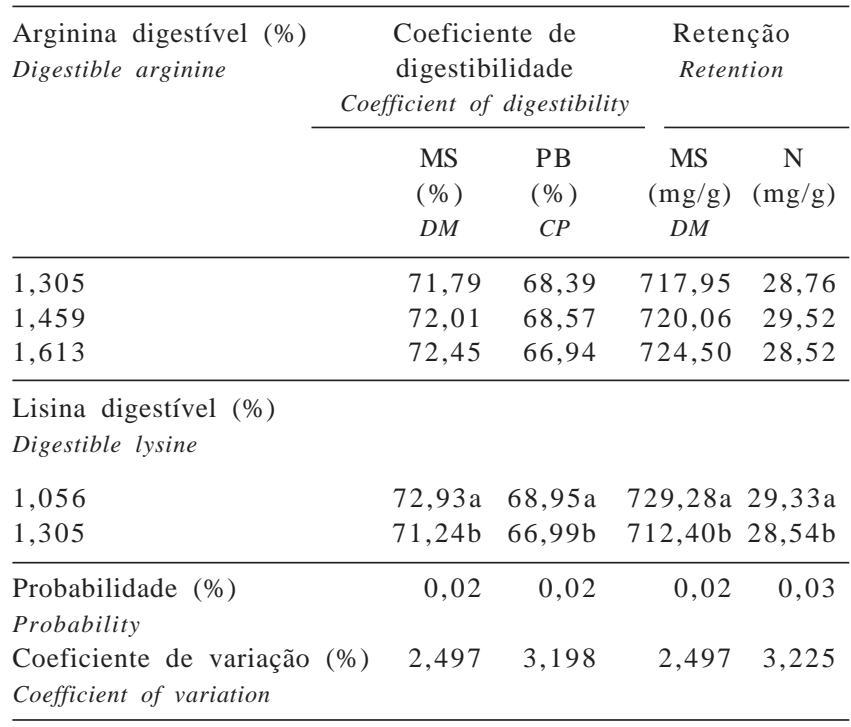

níveis semelhantes aos recomendados pelo NRC (1994) para a fase inicial $(1,25 \%)$ são suficientes para suportar p bom crescimento e manter as funções imunológicas da ave normais. Os resultados deste experimento foram superiores também aos obtidos por Labadan Jr. et al. (2001), que concluíram que os níveis de 1,27 a 1,32\% de arginina digestível proporcionaram máximo desenvolvimento do músculo de peito nas primeiras semanas de vida. 
Tabela 7 - Equações de regressão obtidas para conversão alimentar (CA), consumo de alimento (CR) e peso relativo de proventrículo + moela (PMoe) em frangos de corte alimentados na fase pré-inicial com diferentes de níveis de arginina e lisina

Table 7 - Regression equation for feed-to-gain ratio (FGR), feed intake (FI) and relative weight of proventriculus + gizzard (PGW) obtained with broilers fed diets with different levels of digestible arginine (Arg) and lysine (Lys) in pre-starter phase

\begin{tabular}{|c|c|c|c|c|c|}
\hline \multirow{2}{*}{$\begin{array}{l}\text { Idade (dias) } \\
\text { Age (days) } \\
7\end{array}$} & \multirow{2}{*}{$\begin{array}{l}\text { Variável } \\
\text { Variable }\end{array}$} & \multicolumn{2}{|c|}{$\begin{array}{c}\text { Equação de regressão } \\
\text { Regression equation }\end{array}$} & \multirow{2}{*}{$\begin{array}{l}\mathrm{R}^{2 *} \\
1,00\end{array}$} & \multirow{2}{*}{$\begin{array}{c}\text { CV (\%) })^{1} \\
10,57\end{array}$} \\
\hline & & $\hat{\mathrm{Y}}=-7,592+0,0117 \mathrm{x}-0,000004 \mathrm{x}^{2}$ & Ponto de máxima $=1,463 \%$ Arg dig & & \\
\hline 14 & PMoe (\%) & $\hat{\mathrm{Y}}=6,9154-0,0016 \mathrm{x}$ & & 0,98 & 10,00 \\
\hline 21 & CA (g/g) & $\hat{\mathrm{Y}}=-6,7291+0,0112 \mathrm{x}-0,000004 \mathrm{x}^{2}$ & Ponto de máxima $=1,400 \%$ Arg dig & 1,00 & 6,62 \\
\hline 21 & CR (g) & $\hat{\mathrm{Y}}=865,905+0,1162 \mathrm{x}$ & & 0,80 & 3,02 \\
\hline
\end{tabular}

* Coeficiente de determinação da equação de regressão (coefficient of determination of regression equation).

${ }^{1}$ Coeficiente de variação (coefficient of variation).

\section{Conclusões}

Os níveis de arginina digestível sugeridos para a fase pré-inicial variam de 1,40 a 1,46\%. A utilização de altos níveis de lisina digestível nessa fase demanda ajustes nos níveis de arginina digestível.

\section{Agradecimento}

Ao CNPq, ao Abatedouro São Salvador e à Ajinomoto Biolatina, pelos recursos financeiros disponibilizados a esse projeto.

\section{Literatura Citada}

AJINOMOTO [1990]. Requerimentos de lisina em aves jovens de acordo com a proteína da dieta e níveis de arginina, São Paulo: Ajinomoto-Biolatina, 1990. 8p. (Relatório de pesquisa, 4). Disponível em: <www.lisina.com.br>. Acesso em: 11/04/06.

AJINOMOTO [2004]. Nível de lisina digestível para as dietas pré-iniciais de pintos de corte. São Paulo: AjinomotoBiolatina, 2004. 4p. (Relatório de pesquisa, 41). Disponível em: <www.lisina.com.br>. Acesso em: 11/04/06.

ARAÚJO, L.F. Nutrição pós-eclosão: aspectos teóricos e práticos. In: SIMPÓSIO SOBRE MANEJO E NUTRIÇÃO DE AVES E SUÍNOS, 2003, Campinas. Anais... Campinas: Colégio Brasileiro de Nutrição Animal, 2003. p.183-210.

BATAL, A.B.; PARSONS, C.M. Effects of age on nutrient digestibility in chicks fed different diets. Poultry Science, v.81, n.9, p.400-407, 2002a.

BATAL, A.B.; PARSONS, C.M. Effects of age on development of digestive organs and performance of chicks fed a corn-soybean meal versus a crystalline amino acid diet. Poultry Science, v.81, n.9, p.1338-41, 2002b.

BATAL, A.B.; PARSONS, C.M. Utilization of different soy products as affected by age in chicks. Poultry Science, v.82, n.3, p.454462, 2003.

CHAMRUSPOLLERT, M.; PESTI, G.M.; BAKALLI, R.I. The influence of labile dietary methyl donors on the arginine requirement of young broiler chicks. Poultry Science, v.81, n.8, p.1142-1148, 2002a.

CHAMRUSPOLLERT, M.; PESTI, G.M.; BAKALLI, R.I. Dietary interrelationships among arginine, methionine, and lysine in young broiler chicks. British Journal of Nutrition, v.88, n.6, p.655-660, 2002b.
CHAMRUSPOLLERT, M.; PESTI, G.M.; BAKALLI, R.I. Chick responses to dietary arginine and methionine levels at different environmental temperatures. British Poultry Science, v.45, n.1, p.93-100, 2004.

CYNOBER, L.; Le BOUCHER, J.; VASSON, M.P. Arginine metabolism in mammals. Journal of Nutritional Biochemistry, v.6, 402-413, 1995.

D’MELLO, J.P.F. Amino acid supplementation of cereal-based diets for non-ruminants. Animal Feed Science and Technology, v.45, n.1, p.1-18, 1993.

DIBNER, J. Nutritional requirements of young poultry. In: MEETING OF ARKANSAS NUTRITION CONFERENCE, 1996, Fayetteville. Proceedings... Fayetteville: Arkansas Poultry Federation, 1996. p.15-27.

EDMONDS, M.S.; PARSONS, C.M.; BAKER, D.H. Limiting amino acids in low-protein corn-soybean meal diets fed to growing chicks. Poultry Science, v.64, n.8, p.1519-1526, 1985.

GADELHA, A.C. Resposta produtiva, immune e desenvolvimento ósseo de frangos de corte alimentados com diferentes relações de arginina e lisina digestíveis, Jaboticabal: Universidade Estadual Paulista, 2004. 171p. Tese (Doutorado em Zootecnia) - Universidade Estadual Paulista, 2004.

HURWITZ, S.; SKLAN, D.; TALPAZ, H. et al. The effect of dietary protein level on the lysine and arginine requirements of growing chickens. Poultry Science, v.77, n.5, p.689-696, 1998.

KIDD, M.T.; PEEBLES, E.D.; WHITMARSH,S.K. et al. Growth and immunity of broiler chicks as affected by dietary arginine. Poultry Science, v.80, n.11, p.1535-1542, 2001.

LABADAN JR., M.C.; HSU, K.N.; AUSTIC R.E. Lysine and arginine requirements of broiler chickens at two- to three-week intervals to eight weeks of age. Poultry Science, v.80, n.5, p.599-606, 2001.

MATTERSON, L.D.; POTTER, L.M.; STUTZ, M.W. et al. The metabolizable energy of feeds ingredients for chickens. Storrs: The Univesity of Connecticut, Agricultural Experiment Station, 1965. 11p. (Research Report).

MENDES, A.A.; WATKINS, S.E.; ENGLAND, J.A. et al. Influence of dietary lysine levels and arginine:lysine ratios on performance of broilers exposed to heat or cold stress during the period of three to six weeks of age. Poultry Science, v.76, n.3, p.472481, 1997.

MORAN JR., E.T. Digestion and absorption of carbohydrates in fowl and events through perinatal development. Journal of Nutrition, v.115, n.2, p.665-71, 1985.

NOY, Y.; SKLAN, D. Nutrient use in chicks during the first week post hatch. Poultry Science, v.81, p.391-399, 2002.

NATIONAL RESEARCH COUNCIL - NRC. Nutrient requirements of poultry. 9.ed. Washington, D.C.: National Academy Press, 1994. 155p.

PENZ JR., A.M.; VIEIRA, S.L. Nutrição na primeira semana. In: CONFERÊNCIA APINCO DE CIÊNCIA E TECNOLOGIA 
AVÍCOLAS, 1998, Campinas. Anais... Campinas: Fundação APINCO de Ciência e Tecnologia Avícolas, 1998. p.121-39.

PENZ, JR., A.M.; RENZ, S.V. Actualización em la nutrición de pollos de engorde. In: CONGRESO LATINOAMERICANO DE AVICUltUrA, 18., 2003, Santa Cruz de la Sierra. Anais... Cochabamba: Asociación Nacional de Avicultores de Bolívia e Asociación Latinoamericana de Avicultura, 2003. p.373-384.

ROSTAGNO, H.S.; ALBINO, L.F.T.; DONZELE, J.L. et al. Tabelas brasileiras para aves e suínos: composição de alimentos e exigências nutricionais. Viçosa, MG: Editora UFV, 2000. 141p.

ROSTAGNO, H.S. Tabelas brasileiras para aves e suínos: composição de alimentos e exigências nutricionais. Viçosa, MG: Editora UFV, 2005. 141p.

SILVA, D.J.; QUEIROZ, A.C. Análise de alimentos (métodos químicos e biológicos). 3.ed. Viçosa, MG: Editora UFV, 2002. $165 p$.

STRINGHINI, J.H.; RESENDE, A.; CAFÉ, M.B. et al. Efeito do peso inicial dos pintos e do período da pré-inicial sobre o desempenho de frangos de corte Revista Brasileira de Zootecnia, v.32, n.2, p.353-360, 2003.

STRINGHINI, J.H.; ANDRADE, M.L.; ANDRADE, L. et al. Desempenho, balanço e retenção de nutrientes e biometria dos órgãos digestivos de frangos de corte alimentados com diferentes níveis de proteína na ração pré-inicial. Revista Brasileira de Zootecnia, v.35, n.6, 2350-2358, 2006.
TOLEDO, R.S.; VARGAS, JR., J.G.; ALBINO, L.F.T. et al. Aspectos práticos da nutrição pós-eclosão: níveis nutricionais utilizados, tipos de ingredientes e granulometria da dieta. In: CONFERÊNCIA APINCO DE CIÊNCIA E TECNOLOGIA AVÍCOLAS, 2001, Campinas. Anais..., Campinas: Fundação APINCO de Ciência e Tecnologia Avícolas, 2001. p.153-167.

UNIVERSIDADE FEDERAL DE VIÇOSA - UFV. SAEG - Sistema de análises estatísticas e genéticas. [Manual do usuário]. Versão 7.1. Viçosa, MG: 1997. 150p.

VALERIO, S.R.; OLIVEIRA, R.F.M.; DONZELE, J.L. et al. Níveis de lisina digestível em rações, em que se manteve ou não a relação aminoacídica, para frangos de corte de 1 a 21 dias de idade, mantidos em estresse por calor. Revista Brasileira de Zootecnia, v.32, n.2, p.361-371, 2003. 